\title{
Exploring model transfer strategies for sentiment analysis in Twitter
}

\author{
Eliseu Guimarães ${ }^{1,2}$, Jonnathan Carvalho ${ }^{3}$, Aline Paes $^{1}$, Alexandre Plastino ${ }^{1}$ \\ ${ }^{1}$ Instituto de Computação - Universidade Federal Fluminense - Brazil \\ ${ }^{2}$ Marinha do Brasil - Brazil \\ ${ }^{3}$ Instituto Federal Fluminense - Brazil \\ eliseuguimaraes@id.uff.br, joncarv@iff.edu.br \\ \{alinepaes, plastino\}eic.uff.br
}

\begin{abstract}
Social media have become trendy environments for communication. Because of that, analyze the sentiment that the user expresses in their social media posts is an important research field. However, detecting polarity in such contents is a challenge, partially because the amount of labeled data to train classifiers is scarce in many situations. This paper explores strategies for reusing a model learned from a source dataset to classify instances in a target dataset. The experiments are conducted with 22 tweets sentiment analysis datasets and approaches based on similarity metrics. The results point out that the size of the source training set plays an essential role in the classifiers' performance when they were applied to the target data.
\end{abstract}

Resumo. As mídias sociais se tornaram um ambiente popular para comunicação. Por isso, analisar o sentimento que o usuário expressa em suas postagens nas redes sociais é um importante campo de pesquisa. No entanto, detectar a polaridade em tais conteúdos é um desafio, em parte porque a quantidade de dados rotulados para treinar classificadores é escassa em muitas situações. Este artigo explora estratégias para reusar um modelo aprendido a partir de conjunto de dados fonte para classificar instâncias em um conjunto de dados de destino. Os experimentos são conduzidos com 22 conjuntos de dados de análise de sentimento em tweets e abordagens baseadas em métricas de similaridade. Os resultados apontam que o tamanho do conjunto de treinamento fonte desempenha um papel essencial no desempenho dos classificadores quando usados para inferir a classe das instâncias alvo.

\section{Introdução}

A análise de sentimentos consiste no estudo computacional de identificar opiniões, sentimentos, emoções, humores e atitudes das pessoas [Liu 2020]. Com o surgimento e popularização das redes sociais, como o Twitter $^{1}$, qualquer pessoa pode expressar livremente suas opiniões e sentimentos a respeito de assuntos variados através de textos curtos, os tweets. Tweets são considerados um desafio para a análise de sentimentos devido às

\footnotetext{
${ }^{1}$ http: / /www.twitter.com
} 
suas características, como uso incorreto da gramática, presença frequente de erros ortográficos, falta de contexto devido à limitação a apenas 280 caracteres, bem como a presença de sarcasmo, ironia subjetividade, entre outros [Martínez-Cámara et al. 2014].

A detecção de polaridade em tweets - objetivo deste estudo - consiste na classificação das opiniões expressas em tweets quanto às suas polaridades, aqui tratadas como positivas ou negativas. Abordagens comumente utilizadas para tratar este problema se baseiam em técnicas de aprendizado de máquina, que visam extrair características de tweets previamente rotulados em um dado domínio para treinar classificadores capazes de determinar a polaridade de novos tweets naquele mesmo domínio [Barbosa and Feng 2010, Dong et al. 2014]. Contudo, nem sempre é possível obter dados rotulados em quantidade suficiente para o treinamento de classificadores que alcancem um bom desempenho preditivo. Isso pode ocorrer tanto pela escassez de dados do domínio de interesse, quanto pelo esforço necessário para rotular manualmente uma grande quantidade de dados, muitas vezes proibitivo.

Uma possível solução para o problema da escassez de dados rotulados em um domínio de interesse é aproveitar um classificador aprendido anteriormente para a mesma tarefa, e adaptá-lo ou reusá-lo no domínio pretendido. Essas soluções são investigadas na área de transferência de aprendizado [Pan and Yang 2010], uma vez que as instâncias do domínio de interesse alvo, em geral, são amostradas a partir de uma distribuição distinta da que originou o domínio fonte de treinamento. Entretanto, mesmo oriundos de distribuições distintas, podem existir conjuntos de dados que são mais promissores para a transferência do que outros. No entanto, a seleção adequada de tais conjuntos de dados é um problema desafiador, para o qual diversos estudos têm sido conduzidos na literatura [Guimarães et al. 2020, Guo et al. 2018, Li et al. 2017, Ruder and Plank 2017, Santos et al. 2019]. Esses estudos incluem a avaliação do uso de métricas de similaridade para selecionar uma base de dados apropriada do domínio-fonte (base-fonte) ou selecionar um subconjunto de instâncias do domínio-fonte para treinar um classificador para a base no domínio-alvo (base-alvo), entre outras investigações.

O trabalho apresentado em [Guimarães et al. 2020] visa selecionar a base-fonte mais apropriada para treinar um classificador para uma determinada base-alvo, no contexto da análise de sentimentos em tweets. Nesse caso, a base-fonte é selecionada por meio da análise de similaridade entre a base-alvo e cada base-fonte candidata avaliada, a partir de um conjunto pré-definido de 21 bases-fonte candidatas rotuladas. Dessa forma, a base-fonte candidata mais similar à base-alvo é selecionada para treinar um classificador, que em seguida é avaliado de acordo com suas habilidades preditivas na base-alvo. Em [Guimarães et al. 2020], são avaliadas quatro métricas de similaridade, mas, no geral, os classificadores com melhor poder preditivo foram aqueles treinados com bases-fonte selecionadas por meio da similaridade de cosseno.

Apesar dos resultados promissores obtidos em [Guimarães et al. 2020], não foi investigada a hipótese da utilização de todos os dados disponíveis, por meio da união de todas as bases-fonte candidatas, por exemplo, para treinar o classificador. Além disso, também não foram exploradas estratégias para selecionar um subconjunto de instâncias das bases-fonte, utilizando critérios de similaridade e dissimilaridade entre as instâncias.

Neste contexto, este artigo apresenta um conjunto de experimentos computaci- 
onais com o objetivo de responder às questões de pesquisa definidas a seguir: Q1 Dado um conjunto de bases-fonte rotuladas, vale a pena selecionar uma delas para treinar um classificador de polaridade para uma base-alvo não-rotulada, como feito em [Guimarães et al. 2020], ou um melhor desempenho preditivo poderia ser obtido se o classificador de polaridade para a base-alvo fosse treinado a partir da união de todas as bases-fonte disponíveis? e $\mathbf{Q 2}$ - Considerando a união de todas as bases-fonte disponíveis, vale a pena selecionar um subconjunto de suas instâncias com base na similaridade em relação às instâncias da base-alvo? Os resultados obtidos indicam que unir diferentes bases-fonte candidatas para compor um conjunto de treinamento mais amplo gera classificadores com maior poder preditivo do que os treinados a partir da seleção de uma única base-fonte.

O restante deste artigo está organizado da seguinte forma. A Seção 2 apresenta os trabalhos relacionados. Na Seção 3, são descritos os experimentos conduzidos neste estudo para responder às questões de pesquisa Q1 e Q2. A Seção 4 apresenta os resultados e os discute. Por fim, na Seção 5, são apresentadas as conclusões e trabalhos futuros.

\section{Trabalhos Relacionados}

Além do trabalho apresentado em [Guimarães et al. 2020], diversos outros têm se dedicado ao estudo de técnicas para composição de um conjunto de treinamento a partir de bases-fonte, com o intuito de aprender um classificador para ser aplicado a uma basealvo [Guo et al. 2018, Li et al. 2017, Ruder and Plank 2017, Santos et al. 2019].

Em [Guo et al. 2018], é proposta uma abordagem do tipo mixture-of-experts, na qual se considera que diferentes bases-fonte estão alinhadas a regiões distintas da basealvo. Uma métrica que relaciona as instâncias da base-alvo com as bases-fonte é aprendida, para ponderar os resultados de classificadores treinados utilizando as bases-fonte. Os resultados do estudo mostram que as acurácias preditivas obtidas usando esse método foram superiores às obtidas utilizando como conjunto de treinamento apenas uma basefonte ou a união de todas as bases-fonte.

Por sua vez, [Li et al. 2017] consideram que as instâncias da base-alvo que estejam mais próximas da base-fonte têm maior probabilidade de serem corretamente classificadas. Desta forma, são atribuídos pesos maiores a essas instâncias e é utilizada uma regularização para assegurar uma propagação suave dos rótulos na base-alvo. Esta abordagem foi aplicada em um conjunto de 12 pares de bases e os resultados de acurácia obtidos foram comparados com nove abordagens estabelecidas em outros estudos, mostrando que essa técnica obteve a melhor posição no ranqueamento.

A abordagem proposta em [Ruder and Plank 2017] utiliza otimização Bayesiana para aprender uma métrica de similaridade de bases, que é definida como uma combinação linear de um conjunto de atributos. Foram utilizadas seis métricas de similaridade entre bases como atributos para esse aprendizado, calculadas considerando três tipos de representações dos dados, além de seis métricas de diversidade aplicadas ao conjunto de treinamento. Os resultados apresentados mostram que as acurácias utilizando métricas de similaridade combinadas com métricas de diversidade apresentam melhor desempenho do que utilizar apenas similaridade ou apenas diversidade, além de superarem os resultados de compor o conjunto de treinamento com seleção aleatória ou utilizando uma métrica específica de reconhecido desempenho. 
Em [Santos et al. 2019], são utilizadas métricas de similaridade para a seleção de bases-fonte em português, com o intuito de treinar classificadores para uma base-alvo de tweets no contexto das eleições presidenciais no Brasil em 2018. Nesse trabalho, são utilizadas abordagens de composição do conjunto de treinamento que incluem mesclar as bases mais semelhantes à base-alvo e mesclar as bases menos semelhantes à base-alvo. Os resultados indicam que utilizar bases-fonte mais semelhantes é vantajoso e, ao mesmo tempo, que a inclusão de bases-fonte menos semelhantes ao conjunto de treinamento deteriora o desempenho do classificador.

Diferentemente do proposto por trabalhos anteriores, este estudo combina: (i) a utilização de uma métrica única para a seleção de dados, o que evita o aprendizado e treinamento de uma métrica; (ii) a seleção de instâncias isoladas, o que permite que a quantidade de instâncias da base-fonte dissimilares à base-alvo seja limitada; (iii) a consideração do uso da dissimilaridade como parte do método de seleção, o que pode ajudar a reduzir o overfitting, trazendo diversidade ao conjunto de treinamento; e (iv) o uso de um amplo conjunto de bases-fonte, o que torna os resultados robustos ao utilizar bases com grande variedade de características.

\section{Metodologia Experimental}

Nesta seção, a metodologia adotada nos experimentos computacionais reportados neste estudo é detalhada. Na Seção 3.1, é apresentada a configuração dos experimentos computacionais e, na Seção 3.2, são detalhados os experimentos conduzidos para responder às questões de pesquisa Q1 (Seção 3.2.1) e Q2 (Seção 3.2.2).

\subsection{Configuração dos Experimentos Computacionais}

Nos experimentos computacionais conduzidos neste estudo, são utilizadas 22 bases de dados de tweets em inglês ${ }^{2}$ [Carvalho and Plastino 2021]. As bases são compostas de tweets que expressam opiniões sobre diversos assuntos e rotuladas quanto às suas polaridades, ou seja, se são opiniões positivas ou negativas. Quanto ao conteúdo, enquanto algumas bases contêm tweets sobre um tema específico, como movie e hobbit (filmes), archeage (jogos) e $O M D$ (política), outras são compostas de tweets com conteúdo mais geral, como Narr, SemEval18 e Vader, por exemplo. Além disso, as bases variam em tamanho (quantidade de tweets) e distribuição de classes, como pode ser observado na Tabela 1.

Tabela 1. Quantidade de tweets positivos (\#pos) e negativos (\#neg), percentual de tweets positivos (\%pos) e total de tweets das bases de dados.

\begin{tabular}{|l|r|r|r|r||l|r|r|r|r|}
\hline Bases de dados & \#pos & \#neg & \%pos & Total & Bases de dados & \#pos & \#neg & $\%$ pos & Total \\
\hline irony & 22 & 43 & $34 \%$ & 65 & sarcasm & 33 & 38 & $46 \%$ & 71 \\
aisopos & 159 & 119 & $57 \%$ & 278 & SemEval15-Fig & 47 & 274 & $15 \%$ & 321 \\
sentiment140 & 182 & 177 & $51 \%$ & 359 & person & 312 & 127 & $71 \%$ & 439 \\
hobbit & 354 & 168 & $68 \%$ & 522 & iphone & 371 & 161 & $70 \%$ & 532 \\
movie & 460 & 101 & $82 \%$ & 561 & sanders & 570 & 654 & $47 \%$ & 1224 \\
Narr & 739 & 488 & $60 \%$ & 1227 & archeage & 724 & 994 & $42 \%$ & 1718 \\
SemEval18 & 865 & 994 & $47 \%$ & 1859 & OMD & 710 & 1196 & $37 \%$ & 1906 \\
HCR & 539 & 1369 & $28 \%$ & 1908 & STS-gold & 632 & 1402 & $31 \%$ & 2034 \\
SentiStrength & 1340 & 949 & $59 \%$ & 2289 & Target-dependent & 1734 & 1733 & $50 \%$ & 3467 \\
Vader & 2897 & 1299 & $69 \%$ & 4196 & SemEval13 & 3183 & 1195 & $73 \%$ & 4378 \\
SemEval17 & 2375 & 3972 & $37 \%$ & 6347 & SemEval16 & 8893 & 3323 & $73 \%$ & 12216 \\
\hline
\end{tabular}

${ }^{2}$ https://github.com/joncarv/air-datasets 
Como pré-processamento dos tweets, todas as menções a usuários foram substituídas pelo token único @user e URLs foram substituídas pelo token http://www.url.com. Todos os tweets foram colocados em letras minúsculas e, então, tokenizados. Como atributos para o treinamento dos classificadores é utilizada a abordagem de word embeddings. A geração dos embeddings dos tweets foi feita calculando, para cada um, a média dos embeddings de seus tokens. Para isso, foi adotado o modelo estático pré-treinado apresentado em [Bravo-Marquez et al. 2016]. Este modelo foi treinado em um conjunto de 10 milhões de tweets com o método Skip-gram [Mikolov et al. 2013] e possui 400 dimensões.

O algoritmo de classificação adotado nos experimentos é o de regressão logística, que obteve bom desempenho preditivo no contexto da análise de sentimentos em tweets em [Carvalho and Plastino 2021]. Nesse caso, foi utilizada a implementação da biblioteca scikit-learn $^{3}$, com o valor máximo de iterações igual a 10.000, de modo a evitar falhas na convergência do algoritmo. Para a avaliação dos classificadores foram adotadas as medidas acurácia e $F_{1}$-measure (ponderada).

\subsection{Descrição dos Experimentos Computacionais}

Esta seção descreve os experimentos conduzidos neste estudo para responder às questões de pesquisa Q1 (Seção 3.2.1) e Q2 (Seção 3.2.2), introduzidas na Seção 1.

\subsubsection{Questão de Pesquisa Q1}

$\mathrm{O}$ experimento descrito nesta seção visa responder à questão de pesquisa, Q1 - Dado um conjunto de bases-fonte rotuladas, vale a pena selecionar uma delas para treinar um classificador de polaridade para uma base-alvo não-rotulada, como feito em [Guimarães et al. 2020], ou um melhor desempenho preditivo poderia ser obtido se o classificador de polaridade para a base-alvo fosse treinado a partir da união de todas as bases-fonte disponíveis?

Este experimento investiga a hipótese de utilização de todos os dados disponíveis em bases-fonte candidatas de diversos domínios para treinar um classificador para uma base-alvo, por meio da união das instâncias dessas bases-fonte. Assim, considerando as 22 bases de dados apresentadas na Seção 3.1, cada uma é tratada uma vez como basealvo e a união das 21 bases restantes é a base-fonte. Dessa forma, um classificador é treinado usando como conjunto de treinamento todos os tweets da base-fonte resultante dessa união. Essa estratégia é denominada Estratégia 21D.

Para cada base-alvo avaliada, o classificador treinado com a união das 21 bases restantes é aplicado à base-alvo e o desempenho preditivo (acurácia e $F_{1}$ ) é comparado ao obtido quando o classificador é treinado com a base-fonte candidata mais similar à base-alvo, obtida por meio da similaridade de cosseno, como reportado em [Guimarães et al. 2020] (Estratégia SC).

Além disso, o desempenho preditivo também é comparado com aquele obtido pelo classificador treinado com a própria base-alvo, após a execução de uma validação cruzada com 10 partições (Estratégia Alvo). No entanto, cabe ressaltar que, para a análise

\footnotetext{
${ }^{3}$ https: / / scikit-learn.org/
} 
experimental que está sendo conduzida neste estudo, considera-se que a base-alvo é nãorotulada. Esse fato impediria o treinamento de um classificador com a base-alvo. De todo modo, essa situação é considerada como um baseline para fins de comparação com o desempenho da estratégia 21D descrita nesta seção.

\subsubsection{Questão de Pesquisa Q2}

Os experimentos descritos nesta seção visam responder à questão de pesquisa, Q2 - Considerando a união de todas as bases-fonte disponíveis, vale a pena selecionar um subconjunto de suas instâncias com base na similaridade em relação às instâncias da base-alvo?

Para estes experimentos, o objetivo é treinar um classificador para a base-alvo utilizando um subconjunto das instâncias do conjunto união das bases-fonte. Nesse caso, para cada base-alvo, o conjunto de bases-fonte é formado pela união das 21 bases-fonte restantes disponíveis. Chamaremos esse conjunto de $C_{\text {all }}$ daqui em diante.

Para selecionar as instâncias de $C_{\text {all }}$ que comporão o conjunto de treinamento $C_{\text {train }} \subset C_{\text {all }}$, duas estratégias de seleção de instâncias são investigadas neste estudo, descritas a seguir.

Estratégia S1: Nesta estratégia, $C_{\text {train }}$ é composto por um percentual $p$ de instâncias oriundas de $C_{\text {all }}$. São explorados diferentes valores de $p$, onde $0<p \leq 100, p \in \mathbb{N}$. Duas formas de seleção são analisadas: (sim) seleção de instâncias similares - seleção das instâncias mais similares à base-alvo, e (dis) seleção de instâncias similares e dissimilares - seleção de instâncias mais similares e de instâncias menos similares à base-alvo, em uma razão de 4:1. Por exemplo, com $p=5$, são selecionadas $4 \%$ das instâncias de $C_{\text {all }}$ que sejam mais similares à base-alvo e $1 \%$ das instâncias menos similares. $\mathrm{O}$ cálculo da similaridade é realizado por meio da similaridade de cosseno, devido ao bom desempenho reportado em [Guimarães et al. 2020].

Para computar a similaridade, a base-alvo será representada por uma única representação vetorial de 400 dimensões. Para isso, duas estratégias de representação são analisadas: (mt) média de tokens - a representação como sendo a média dos embeddings de todos os tokens que compõem a base, e (mi) média de instâncias - a representação como sendo a média dos embeddings das instâncias pertencentes à base.

Com relação à distribuição de classes dos conjuntos de treinamento $C_{\text {train }}$, duas situações são analisadas: (i) sem balanceamento - seleção de instâncias mantendo a distribuição original de classes de $C_{\text {all }}$, e (ii) com balanceamento - seleção de instâncias com uma distribuição balanceada. Nesse caso, para a estratégia de seleção sim, as instâncias da classe majoritária menos similares à base-alvo não são selecionadas. Por outro lado, para a estratégia de seleção dis, como são selecionadas as mais similares e as menos similares em uma razão de 4:1, as instâncias intermediárias da classe majoritária não são selecionadas. Por exemplo, se 700 instâncias pertencem à classe majoritária e 500 à classe minoritária, quando $p=100$, são selecionadas, da classe majoritária, as 400 instâncias mais similares à base-alvo e as 100 menos similares $(400+100=500$, que é a quantidade de instâncias da classe minoritária).

Estratégia S2: Nesta estratégia, para cada base-alvo avaliada, o conjunto de treinamento $C_{\text {train }}$, é formado selecionando-se, para cada instância da base-alvo, as $k$ instâncias de $C_{\text {all }}$ 
mais similares a ela. A seleção é feita de maneira iterativa, como descrito a seguir. $\mathrm{Na}$ primeira iteração, a instância em $C_{\text {all }}$ mais similar a cada instância da base-alvo é selecionada. Na próxima iteração, a segunda instância mais similar a cada instância da base-alvo é selecionada e, assim, sucessivamente até a $k$-ésima iteração. Este procedimento assegura que, para dois valores $i$ e $j$, tais que $k_{i}<k_{j}$, cada conjunto de treinamento $C_{\text {train }}$ gerado obedece à relação $C_{\text {train }_{i}} \subset C_{\text {train }_{j}}$.

Para avaliar as estratégias S1 e S2, os classificadores obtidos a partir dos conjuntos $C_{\text {train }}$ são aplicados à base-alvo e o desempenho preditivo (acurácia e $F_{1}$ ) é comparado com aquele obtido pelo classificador treinado com as instâncias da própria base-alvo, após a execução de uma validação cruzada com 10 partições e com os resultados obtidos pela Estratégia 21D.

\section{Resultados Experimentais}

Nesta seção, são reportados os resultados dos experimentos para responder às questões de pesquisa Q1 (Seção 4.1 e Q2 (Seção 4.2).

\subsection{Respondendo à Questão de Pesquisa Q1}

A Tabela 2 apresenta os resultados obtidos para responder à questão de pesquisa Q1. A segunda, terceira e quarta colunas apresentam as acurácias preditivas dos classificadores treinados com a própria base-alvo (estratégia Alvo), com a base-fonte selecionada pela similaridade de cosseno (estratégia SC), reportados em [Guimarães et al. 2020], e com a base formada pela união das 21 bases-fonte (estratégia 21D). De forma semelhante, a sétima, oitava e nona colunas apresentam os valores de $F_{1}$. Os melhores resultados encontram-se sublinhados. Observando os valores registrados na tabela, a estratégia $21 \mathrm{D}$ apresenta melhores resultados que a estratégia Alvo em 16 das 22 bases-alvo em termos de acurácia, e em 17 das 22 bases-alvo em termos de $F_{1}$.

Além dos desempenhos preditivos das estratégias avaliadas, também são analisados os ganhos obtidos ao treinar um classificador com determinada estratégia (SC ou 21D), em relação a utilizar a própria base-alvo como conjunto de treinamento. Nesse caso, valores de ganho maiores que 1 significam que treinar um classificador com a estratégia avaliada produz um desempenho melhor do que utilizar a base-alvo.

$\mathrm{Na}$ Tabela 2, a quinta coluna apresenta, em termos de acurácia, os valores dos ganhos obtidos comparando o desempenho da estratégia SC [Guimarães et al. 2020] com o desempenho da estratégia Alvo (coluna SC $x$ Alvo). A sexta coluna indica os ganhos de acurácia obtidos comparando o desempenho da estrategia 21D com o desempenho da estratégia Alvo (coluna 21D $x$ Alvo). De forma semelhante, na décima e na décimaprimeira colunas, os ganhos apresentados são referentes aos resultados obtidos em termos de $F_{1}$. Por fim, em negrito encontram-se assinalados os maiores valores de ganho.

Analisando os ganhos obtidos pelas estratégias avaliadas, é possível observar que treinar um classificador usando a união de todas as bases-fonte candidatas disponíveis (colunas 21D $x$ Alvo) gera um resultado melhor do que treinar um classificador com uma única base-fonte selecionada pela similaridade de cosseno (colunas SC x Alvo), para 20 das 22 bases-alvo, tanto para acurácia quanto para $F_{1}$. Além disso, considerando os valores médios dos ganhos obtidos, apresentados na última linha (Ganho médio), é possível notar um aumento considerável tanto para acurácia (de 0,92 para 1,03 ) quanto para $F_{1}$ 
Tabela 2. Análise de desempenho dos classificadores treinados com a união das bases-fonte disponíveis.

\begin{tabular}{|c|c|c|c|c|c|c|c|c|c|c|}
\hline \multirow{3}{*}{ Bases de dados } & \multicolumn{5}{|c|}{ Acurácia } & \multicolumn{5}{|c|}{$F_{1}$-measure } \\
\hline & \multirow{2}{*}{ Alvo } & \multirow{2}{*}{$\mathrm{SC}^{*}$} & \multirow{2}{*}{$21 \mathrm{D}$} & \multicolumn{2}{|c|}{ Ganho } & \multirow{2}{*}{ Alvo } & \multirow{2}{*}{$\mathrm{SC}^{*}$} & \multirow{2}{*}{$21 \mathrm{D}$} & \multicolumn{2}{|c|}{ Ganho } \\
\hline & & & & SC x Alvo & 21D x Alvo & & & & SC x Alvo & 21D x Alvo \\
\hline irony & 0,66 & 0,68 & 0,77 & 1,02 & 1,16 & 0,53 & 0,68 & 0,76 & 1,30 & 1,45 \\
\hline sarcasm & 0,56 & 0,58 & $\overline{0,76}$ & 1,02 & 1,35 & 0,43 & 0,53 & $\overline{0,76}$ & 1,24 & 1,78 \\
\hline aisopos & 0,81 & 0,86 & $\overline{0,88}$ & 1,06 & 1,08 & 0,80 & 0,86 & $\overline{0,88}$ & 1,07 & 1,10 \\
\hline SemEval15-Fig & 0,85 & 0,70 & $\overline{0,59}$ & $\mathbf{0 , 8 2}$ & 0,69 & 0,79 & 0,74 & $\overline{0,65}$ & 0,94 & 0,83 \\
\hline sentiment140 & $\overline{0,81}$ & 0,69 & 0,86 & 0,85 & 1,07 & $\overline{0,80}$ & 0,67 & 0,86 & 0,83 & 1,07 \\
\hline person & 0,71 & 0,73 & $\overline{0,81}$ & 1,03 & 1,13 & 0,59 & 0,71 & $\overline{0,80}$ & 1,19 & 1,35 \\
\hline hobbit & 0,68 & 0,69 & $\overline{0,75}$ & 1,02 & 1,11 & 0,55 & 0,64 & $\overline{0,74}$ & 1,16 & 1,35 \\
\hline iphone & 0,70 & 0,71 & $\underline{0,74}$ & 1,02 & 1,06 & 0,57 & 0,72 & $\underline{0,75}$ & 1,26 & 1,31 \\
\hline movie & 0,82 & 0,81 & $\overline{0,83}$ & 0,99 & 1,01 & 0,74 & 0,78 & $\overline{0,83}$ & 1,06 & 1,12 \\
\hline sanders & 0,76 & 0,61 & $\overline{0,77}$ & 0,80 & 1,01 & 0,76 & 0,57 & $\overline{0,77}$ & 0,76 & 1,02 \\
\hline Narr & 0,83 & 0,66 & $\overline{0,89}$ & 0,79 & 1,07 & 0,83 & 0,64 & $\overline{0,89}$ & 0,78 & 1,08 \\
\hline archeage & $\underline{0,82}$ & 0,57 & $\overline{0,77}$ & 0,70 & 0,93 & $\underline{0,82}$ & 0,54 & $\overline{0,77}$ & 0,66 & 0,94 \\
\hline SemEval18 & $\overline{0,77}$ & 0,63 & $\underline{0,81}$ & 0,82 & 1,05 & $\overline{0,77}$ & 0,60 & $\underline{0,81}$ & 0,78 & 1,05 \\
\hline OMD & $\underline{0,76}$ & 0,65 & $\overline{0,71}$ & 0,85 & 0,94 & $\underline{0,73}$ & 0,65 & $\overline{0,69}$ & 0,90 & 0,95 \\
\hline HCR & 0,72 & 0,73 & $\underline{0,74}$ & 1,01 & $\mathbf{1 , 0 3}$ & 0,60 & 0,62 & 0,67 & 1,04 & 1,11 \\
\hline STS-gold & 0,78 & 0,80 & 0,71 & 1,03 & 0,91 & 0,75 & 0,80 & 0,72 & 1,07 & 0,97 \\
\hline SentiStrength & 0,75 & $\overline{0,71}$ & 0,79 & 0,94 & 1,05 & 0,74 & $\overline{0,67}$ & 0,79 & 0,90 & 1,06 \\
\hline Target-dependent & $\underline{0,80}$ & 0,66 & $\overline{0,77}$ & 0,83 & $\mathbf{0 , 9 7}$ & $\underline{0,80}$ & 0,65 & $\overline{0,77}$ & 0,82 & 0,97 \\
\hline Vader & $\overline{0,83}$ & 0,81 & $\underline{0,84}$ & 0,98 & 1,01 & $\overline{0,81}$ & 0,81 & $\underline{0,84}$ & 1,00 & 1,04 \\
\hline SemEval13 & 0,77 & 0,77 & $\overline{0,83}$ & 1,01 & 1,08 & 0,71 & 0,73 & $\overline{0,81}$ & 1,03 & 1,15 \\
\hline SemEval17 & $\underline{0,85}$ & 0,62 & $\overline{0,85}$ & 0,72 & 0,99 & 0,85 & 0,60 & $\overline{0,85}$ & 0,71 & 1,00 \\
\hline \multirow[t]{2}{*}{ SemEval16 } & 0,82 & 0,80 & 0,84 & 0,97 & 1,02 & 0,81 & 0,77 & 0,84 & 0,96 & 1,04 \\
\hline & \multicolumn{3}{|c|}{ Ganho médio: } & 0,92 & 1,03 & \multicolumn{3}{|c|}{ Ganho médio: } & 0,97 & 1,13 \\
\hline
\end{tabular}

(de 0,97 para 1,13) ao utilizar a união de todas as bases-fonte candidatas, em detrimento a selecionar uma base-fonte específica.

\subsection{Respondendo à Questão de Pesquisa Q2}

Esta seção apresenta as avaliações das estratégias de seleção de instâncias, S1 e S2, descritas na Seção 3.2.2, para responder à questão de pesquisa Q2.

\subsubsection{Estratégia de Seleção S1}

A estratégia $\mathrm{S} 1$ consiste na seleção de um percentual $p$ das instâncias da base-fonte. Na Tabela 3, os resultados reportados correspondem aos valores de ganho médio obtidos ao avaliar as possíveis combinações da representação da base-alvo com a forma de seleção de instâncias. Mais especificamente, as formas de representação da base-alvo, $m t$ (utilizando a média dos embeddings dos tokens) e mi (utilizando a média dos embeddings das instâncias), são combinadas com as formas de seleção de instâncias, sim (selecionando apenas as instâncias mais similares à base-alvo) e dis (selecionando as mais similares e as mais dissimilares), a saber: $\boldsymbol{m} \boldsymbol{t}+\boldsymbol{s i m}, \boldsymbol{m} \boldsymbol{t}+\boldsymbol{d i s}, \boldsymbol{m i}+\boldsymbol{s i m}$ e $\boldsymbol{m i}+\boldsymbol{d i s}$. O ganho médio consiste na média dos ganhos para as 22 bases de dados avaliadas. Devido ao espaço limitado, para cada combinação avaliada, são reportados apenas os resultados dos cinco subconjuntos $C_{\text {train }}$ que obtiveram os melhores ganhos médios.

A parte esquerda da Tabela 3 apresenta a avaliação do cenário em que o percentual selecionado segue a distribuição original de classes da base-fonte (sem balanceamento) e na parte direita estão os resultados obtidos quando são selecionadas quantidades iguais de instâncias positivas e negativas (com balanceamento). Os resultados em negrito indicam os casos em que os ganhos médios são superiores aos obtidos ao utilizar toda a base-fonte, como reportado na Tabela 2 (1,03 e 1,13, em termos de acurácia e $F_{1}$, respectivamente). 
Considerando a combinação $m t+\operatorname{sim}$ para os casos sem balanceamento, é possível observar que apenas um subconjunto - $\operatorname{com} p=99$ - obteve desempenho superior do que usar $C_{\text {all }}$ como conjunto de treinamento, tanto para acurácia quanto para $F_{1}$. Nas situações em que o conjunto de treinamento é balanceado, nenhum subconjunto obteve resultados melhores de quando $C_{\text {all }}$ é o conjunto de treinamento.

Analisando a configuração $m t+d i s$, isto é, utilizando a média dos embeddings dos tokens $(m t)$ para representação da base-alvo e selecionando as instâncias mais similares e as mais dissimilares (dis), para as situações sem balanceamento, é possível notar que quatro subconjuntos - quando $p=99, p=95, p=96, p=94$ - obtiveram desempenho melhor do usar $C_{\text {all }}$ como treinamento, tanto para acurácia quanto para $F_{1}$. Nos casos com balanceamento, todos os cinco melhores subconjuntos - quando $p=94, p=98$, $p=97, p=95$, e $p=93$ - apresentaram desempenhos superiores do que usar $C_{\text {all }}$ como treinamento, em termos de acurácia, e nos subconjuntos quando $p=98, p=94, p=97$, $p=95$ e $p=100$, em termos de $F_{1}$.

Tabela 3. Subconjuntos da base-fonte (\%) com melhores desempenhos

\begin{tabular}{|c|c|c|c|c|c|c|c|c|c|}
\hline \multicolumn{5}{|c|}{ Sem balanceamento } & \multicolumn{5}{|c|}{ Com balanceamento } \\
\hline \multirow{2}{*}{ Pos. } & \multicolumn{2}{|c|}{ Acurácia } & \multicolumn{2}{|c|}{$F_{1}$-measure } & \multirow{2}{*}{ Pos. } & \multicolumn{2}{|c|}{ Acurácia } & \multicolumn{2}{|c|}{$F_{1}$-measure } \\
\hline & $\%$ sel. & Ganho & $\%$ sel. & Ganho & & $\%$ sel. & Ganho & $\%$ sel. & Ganho \\
\hline \multicolumn{10}{|c|}{ Combinação $m t+\operatorname{sim}$} \\
\hline $1^{\circ}$ & $99 \%$ & $\mathbf{1 , 0 3 3 8}$ & $99 \%$ & $\mathbf{1 , 1 2 6 1}$ & $1^{\circ}$ & $66 \%$ & 1,0311 & $95 \%$ & 1,1224 \\
\hline $2^{\circ}$ & $100 \%$ & 0332 & $100 \%$ & 1,1253 & $2^{\circ}$ & $65 \%$ & 1,0311 & $98 \%$ & 1,1223 \\
\hline $3^{\circ}$ & $96 \%$ & 0329 & $96 \%$ & 1,1249 & $3^{\circ}$ & $95 \%$ & 0311 & $84 \%$ & 1222 \\
\hline $4^{\circ}$ & $97 \%$ & 1,03 & $98 \%$ & 1,1243 & $4^{\circ}$ & $85 \%$ & 1,0311 & $97 \%$ & 1,1222 \\
\hline $5^{\circ}$ & & & $97 \%$ & 1,1243 & $5^{\circ}$ & $98 \%$ & & $96 \%$ & 1,1221 \\
\hline \multicolumn{10}{|c|}{ Combinação $m t+d i s$} \\
\hline $1^{\circ}$ & $99 \%$ & 1,0340 & $99 \%$ & $\mathbf{1 , 1 2 6 5}$ & $1^{\circ}$ & $94 \%$ & & $98 \%$ & 1,1290 \\
\hline $2^{\circ}$ & $95 \%$ & 1, & $95 \%$ & 1,1258 & $2^{\circ}$ & $98 \%$ & & $94 \%$ & $\overline{\mathbf{1 , 1 2 9 0}}$ \\
\hline $3^{\circ}$ & $96 \%$ & & $96 \%$ & & $3^{\circ}$ & $97 \%$ & & $97 \%$ & $\overline{1,1287}$ \\
\hline $4^{\circ}$ & $94 \%$ & & $94 \%$ & & $4^{\circ}$ & $95 \%$ & & $95 \%$ & 1,1285 \\
\hline $5^{\circ}$ & $100 \%$ & & $93 \%$ & 1,1253 & $5^{\circ}$ & $93 \%$ & & $100 \%$ & 1,1284 \\
\hline \multicolumn{10}{|c|}{ Combinação mi+sim } \\
\hline $1^{\circ}$ & $99 \%$ & 1 & $99 \%$ & 1,1257 & $1^{\circ}$ & $86 \%$ & 1,03 & $98 \%$ & 1,1225 \\
\hline $2^{\circ}$ & $100 \%$ & & $100 \%$ & & $2^{\circ}$ & $87 \%$ & & $86 \%$ & 1225 \\
\hline $3^{\circ}$ & $93 \%$ & & $93 \%$ & & $3^{\circ}$ & $85 \%$ & & $99 \%$ & 1,1224 \\
\hline $4^{\circ}$ & $2 \%$ & & $96 \%$ & & $4^{\circ}$ & $84 \%$ & & $87 \%$ & 1,1224 \\
\hline $5^{\circ}$ & $96 \%$ & 1,0329 & $98 \%$ & 1,1247 & $5^{\circ}$ & $73 \%$ & & $94 \%$ & 1,1224 \\
\hline \multicolumn{10}{|c|}{ Combinação $m i+d i s$} \\
\hline $1^{\circ}$ & $93 \%$ & & $93 \%$ & 1,1266 & $1^{\circ}$ & $98 \%$ & 1,0368 & $98 \%$ & 1,1289 \\
\hline $2^{\circ}$ & $91 \%$ & 1, & $91 \%$ & 1262 & $2^{\circ}$ & $94 \%$ & 367 & $94 \%$ & 1,1287 \\
\hline $3^{\circ}$ & $92 \%$ & & $92 \%$ & & $3^{\circ}$ & $99 \%$ & & $100 \%$ & 1,1287 \\
\hline $4^{\circ}$ & $100 \%$ & & $89 \%$ & & $4^{\circ}$ & $100 \%$ & & $99 \%$ & 1,1286 \\
\hline $5^{\circ}$ & $94 \%$ & 1,0332 & $100 \%$ & 1,1253 & $5^{\circ}$ & $92 \%$ & 1,0364 & $96 \%$ & 1,1284 \\
\hline
\end{tabular}

Quanto à configuração $m i+\operatorname{sim}$, apenas um subconjunto - com $p=99$, sem balanceamento - apresenta ganho superior ao obtido com o treinamento realizado com $C_{a l l}$, tanto para acurácia quanto para $F_{1}$.

Por último, para a configuração $m i+d i s$, analisando os casos sem balanceamento, três subconjuntos $-\operatorname{com} p=93, p=91$ e $p=92-$ apresentam resultados melhores do que usar $C_{\text {all }}$ como treinamento, tanto para acurácia quanto para $F_{1}$. No entanto, em termos de $F_{1}$, o subconjunto formado por $p=89$ das instâncias também apresentou desempenho superior. Para os casos com balanceamento, os cinco melhores subconjuntos - com $p=98, p=94, p=99, p=100$ e $p=92$, em termos de acurácia e $p=98$, $p=94, p=100, p=99$ e $p=96$ em termos de $F_{1}$ - apresentam desempenho superior ao obtido utilizando $C_{\text {all }}$ como treinamento.

$\mathrm{Na}$ avaliação geral, considerando os maiores entre todos os valores de ganho 
médio obtidos (valores sublinhados), é possível notar que a configuração $m t+$ dis com balanceamento apresentou os melhores resultados. Especificamente, em termos de acurácia, quando $p=94$, obteve-se ganho de 1,0370 e, em termos de $F_{1}$, com $p=94$ e $p=98$ foi obtido um ganho de 1,1290. No entanto, cabe destacar que os resultados reportados na Tabela 3, que correspondem aos subconjuntos da base-fonte com melhores desempenhos, foram obtidos com percentuais muito próximos a 100\%, dando evidências de que essa estratégia de seleção de instâncias não foi efetiva.

\subsubsection{Estratégia de Seleção S2}

A estratégia S2 consiste na seleção das $k$ instâncias da base-fonte mais similares a cada instância da base-alvo. Devido ao espaço limitado, são reportados apenas os melhores resultados, obtidos com o balanceamento da base-fonte, considerando que essa situação apresentou o melhor desempenho geral. A Tabela 4 apresenta os melhores resultados obtidos para cada base-alvo, variando o valor de $k$ entre 1 e 20 . Especificamente, para cada instância da base-alvo, são selecionadas as $k$ instâncias da base-fonte mais similares a cada uma delas, tal que $1 \leq k \leq \min \left(k_{\max }, 20\right)$, em que $k_{\max }$ é definido a seguir.

O número de instâncias da base-fonte de cada classe a serem selecionadas, $n_{\text {sel }}$, para um determinado $k$, é dado por $n_{\text {sel }}=\left(k \times n_{\text {alvo }}\right) / 2$, em que $n_{\text {alvo }}$ é o número de instâncias da base-alvo. Dessa forma, sabendo que $n_{\text {sel }} \leq n_{\min }$, em que $n_{\min }$ é o número de instâncias que pertencem à classe minoritária na base-fonte, temos que $k \leq\left(2 \times n_{\min }\right) / n_{\text {alvo }}$. Logo, o valor máximo para $k, k_{\max }$, é definido por $k_{\max }=$ $\left\lfloor\left(2 \times n_{\text {min }}\right) / n_{\text {alvo }}\right\rfloor$.

Tabela 4. Valores de $\mathbf{k}$ com melhores desempenhos.

\begin{tabular}{|c|c|c|c|c|c|}
\hline \multirow{2}{*}{ Bases de dados } & \multirow{2}{*}{$k_{\max }$} & \multicolumn{2}{|c|}{ Acurácia (ganho) } & \multicolumn{2}{|c|}{$F_{1}$-measure (ganho) } \\
\hline & & Melhor $k$ x Alvo & 21D x Alvo & Melhor $k$ x Alvo & 21D x Alvo \\
\hline irony & 20 & $20(1,0692)$ & 1,1621 & $20(1,3528)$ & 1,4472 \\
\hline sarcasm & 20 & $2(1,3479)$ & 1,3479 & $10(\mathbf{1 , 7 8 2 6})$ & 1,7814 \\
\hline aisopos & 20 & $6(\mathbf{1 , 0 8 9 0})$ & 1,0845 & $6(\mathbf{1}, \mathbf{1 0 8 3})$ & 1,1019 \\
\hline SemEval15-Fig & 20 & $5(\mathbf{0 , 7 0 8 1})$ & 0,6934 & $5(\mathbf{0 , 8 4 2 4})$ & 0,8287 \\
\hline sentiment 140 & 20 & $17(1,0518)$ & 1,0691 & $17(1,0536)$ & 1,0709 \\
\hline person & 20 & $20(1,0930)$ & 1,1347 & $20(1,3227)$ & 1,3549 \\
\hline hobbit & 20 & $20(\mathbf{1 , 1 1 0 1})$ & 1,1073 & $20(1,3457)$ & 1,3516 \\
\hline iphone & 20 & $14(1,0295)$ & 1,0619 & $14(1,2727)$ & 1,3102 \\
\hline movie & 20 & $16(0,9891)$ & 1,0065 & $16(1,1056)$ & 1,1228 \\
\hline sanders & 20 & $15(\mathbf{1 , 0 2 4 7 )}$ & 1,0141 & $15(\mathbf{1 , 0 3 3 8})$ & 1,0229 \\
\hline Narr & 20 & $20(1,0589)$ & 1,0716 & $20(1,0660)$ & 1,0774 \\
\hline archeage & 20 & $4(\mathbf{0 , 9 7 3 7 )}$ & 0,9348 & $4(\mathbf{0 , 9 8 0 6})$ & 0,9438 \\
\hline SemEval18 & 20 & $16(\mathbf{1 , 0 5 2 8})$ & 1,0452 & $16(\mathbf{1 , 0 6 0 5})$ & 1,0533 \\
\hline OMD & 20 & $19(\mathbf{0 , 9 4 5 4 )}$ & 0,9411 & $19(\mathbf{0 , 9 5 0 6})$ & 0,9505 \\
\hline HCR & 20 & $18(\mathbf{1 , 0 3 7 2})$ & 1,0300 & $2(\mathbf{1 , 1 6 6 9 )}$ & 1,1132 \\
\hline STS-gold & 19 & $2(\mathbf{0 , 9 8 9 9})$ & 0,9109 & $2(1,0492)$ & 0,9680 \\
\hline SentiStrength & 17 & $13(\mathbf{1 , 0 5 1 9 )}$ & 1,0478 & $13(\mathbf{1 , 0 6 1 6})$ & 1,0564 \\
\hline Target-dependent & 10 & $6(0,9746)$ & 0,9659 & $6(0,9747)$ & 0,9657 \\
\hline Vader & 8 & $8(1,0041)$ & 1,0121 & $8(1,0324)$ & 1,0399 \\
\hline SemEval13 & 8 & $8(1,0629)$ & 1,0781 & $6(1,1425)$ & 1,1527 \\
\hline SemEval17 & 5 & $2(\mathbf{1 , 0 0 2 8})$ & 0,9947 & $2(1,0079)$ & 1,0014 \\
\hline SemEval16 & 2 & $2(1,0118)$ & $\mathbf{1 , 0 1 7 7}$ & $2(1,0372)$ & 1,0424 \\
\hline
\end{tabular}

$\mathrm{Na}$ Tabela 4, a segunda coluna apresenta os valores máximos de $k$ para cada basealvo. A terceira e quinta colunas indicam os valores de $k$ que produziram os melhores ganhos, em termos de acurácia e $F_{1}$, respectivamente, em relação ao desempenho obtido com o classificador treinado com a própria base-alvo. Entre parênteses são apresentados os valores desses ganhos. A quarta e sexta colunas apresentam os valores dos ganhos 
obtidos ao usar toda a base-fonte para treinamento (21D), em termos de acurácia e $F_{1}$, respectivamente. Em negrito estão destacados os melhores valores de ganho.

Ao analisar os ganhos reportados na Tabela 4, é possível observar que a estratégia de seleção de instâncias S2 apresentou melhor desempenho em 12 das 22 bases (colunas Melhor $k x$ Alvo), tanto para acurácia quanto para $F_{1}$, em relação ao desempenho utilizando toda a base-fonte (colunas 21D $x$ Alvo). Além disso, os valores de $k$ que produzem os melhores resultados estão entre os mais próximos de $k_{\max }$. Analisando as acurácias, para sete bases-alvo, o melhor valor de $k$ é igual a $k_{\max }$ e para outras duas bases, OMD e HCR, o melhor valor de $k$ se aproxima a $k_{\max }\left(k \geq k_{\max }-2\right)$. Em termos de $F_{1}$, para seis bases o melhor $k$ é igual a $k_{\max }$ e para outras duas, OMD e SemEval13, o melhor desempenho também acontece para $k \geq k_{\max }-2$.

Assim como para os resultados obtidos pela estratégia de seleção S1 (Seção 4.2.1), é possível notar uma tendência de aumento no desempenho preditivo da estratégia S2 quanto maior é o conjunto de treinamento, dando evidências de que este tipo de seleção também não foi efetiva.

\section{Conclusões e Trabalhos Futuros}

Este artigo teve como objetivo determinar se vale a pena treinar um classificador de polaridade com a união de um conjunto de bases-fonte disponíveis, ou se é melhor selecionar uma base-fonte específica (Q1), como feito em [Guimarães et al. 2020], e para verificar se vale a pena selecionar um subconjunto de instâncias da união das bases-fonte disponíveis, com base na similaridade em relação às instâncias da base-alvo (Q2).

Para responder Q1, o experimento realizado apontou que usar todas as bases como fonte para o treinamento do classificador alcança, em geral, melhores resultados que selecionar uma única base, uma vez que o ganho médio obtido foi superior ao observado em [Guimarães et al. 2020].

Para responder Q2, duas formas de seleção de instâncias foram analisadas: selecionando um percentual das instâncias da base-fonte (estratégia S1) ou as $k$ instâncias mais similares a cada instância da base-alvo (estratégia $\mathrm{S} 2$ ). Em relação à estratégia $\mathrm{S} 1$, foi possível notar que a seleção de instâncias da base-fonte para compor o conjunto de treinamento pode gerar classificadores com um ganho médio melhor do que o ganho obtido ao usar toda a base-fonte, porém com resultados muito próximos. No entanto, os percentuais que devem ser selecionados são, em sua maioria, elevados (próximos a 100\%), não compensando o custo computacional de selecionar as instâncias. As configurações que obtiveram o melhor desempenho neste experimento foram as que selecionavam as instâncias mais similares e as mais dissimilares $(m t+d i s$ e $m i+d i s)$, e consideravam o balanceamento da base-fonte. Isso indica que a diversidade e o balanceamento do conjunto de treinamento podem influenciar positivamente no desempenho do classificador.

Quanto à estratégia S2, mais uma vez houve a indicação de que, ao aumentar o conjunto de treinamento, o desempenho do classificador tende a melhorar. Embora nesta avaliação existam situações em que o desempenho da seleção tenha apresentado melhores resultados do que usar toda a base-fonte, isso ocorre com uma pequena diferença de desempenho na maioria dos casos. Contudo, como para algumas bases o melhor desempenho foi obtido com $k=20$, é possível obter um desempenho melhor ao aumentar o valor de $k$. 
Em trabalhos futuros, podem ser exploradas outras métricas de distância para seleção de instâncias e outras formas de representações dos dados. Tendo em vista que incluir instâncias dissimilares mostrou-se promissor, novos trabalhos podem focar em ajustar a razão entre instâncias similares e dissimilares que estão sendo utilizadas.

\section{Referências}

Barbosa, L. and Feng, J. (2010). Robust sentiment detection on twitter from biased and noisy data. In Proc. of the 23rd Int. Conf. on Computational Linguistics: Posters, COLING'10, page 36-44. ACL.

Bravo-Marquez, F., Frank, E., Mohammad, S. M., and Pfahringer, B. (2016). Determining word-emotion associations from tweets by multi-label classification. In Proc. of the 2016 IEEE/WIC/ACM International Conference on Web Intelligence (WI), pages 536539. IEEE.

Carvalho, J. and Plastino, A. (2021). On the evaluation and combination of state-of-the-art features in twitter sentiment analysis. Artificial Intelligence Review, 54.

Dong, L., Wei, F., Tan, C., Tang, D., Zhou, M., and Xu, K. (2014). Adaptive recursive neural network for target-dependent Twitter sentiment classification. In Proc. of the 52nd Annual Meeting of the Association for Computational Linguistics (Volume 2: Short Papers), pages 49-54. ACL.

Guimarães, E., Carvalho, J., Paes, A., and Plastino, A. (2020). Transfer learning for twitter sentiment analysis: Choosing an effective source dataset. In Anais do VIII Symposium on Knowledge Discovery, Mining and Learning, pages 161-168. SBC.

Guo, J., Shah, D., and Barzilay, R. (2018). Multi-source domain adaptation with mixture of experts. In Proc. of the 2018 Conference on Empirical Methods in Natural Language Processing, pages 4694-4703. ACL.

Li, S., Song, S., and Huang, G. (2017). Prediction reweighting for domain adaptation. IEEE Transactions on Neural Networks and Learning Systems, 28(7):1682-1695.

Liu, B. (2020). Sentiment Analysis: Mining Opinions, Sentiments, and Emotions. Studies in Natural Language Processing. Cambridge University Press, 2 edition.

Martínez-Cámara, E., Martín-Valdivia, M., López, L., and Montejo-Ráez, A. (2014). Sentiment analysis in twitter. Natural Language Engineering, 20:1-28.

Mikolov, T., Chen, K., Corrado, G. S., and Dean, J. (2013). Efficient Estimation of Word Representations in Vector Space. CoRR, abs/1301.3781.

Pan, S. J. and Yang, Q. (2010). A Survey on Transfer Learning. IEEE Transactions on Knowledge and Data Engineering, 22(10):1345-1359.

Ruder, S. and Plank, B. (2017). Learning to select data for transfer learning with Bayesian Optimization. In Proc. of the 2017 Conference on Empirical Methods in Natural Language Processing, pages 372-382. ACL.

Santos, J. S., Paes, A., and Bernardini, F. (2019). Combining labeled datasets for sentiment analysis from different domains based on dataset similarity to predict electors sentiment. In 2019 8th Brazilian Conf. on Intelligent Systems, pages 455-460. 